*Correspondencia:

realcottoi@gmail.com

Departamento de Bioestadística, Instituto Oncológico Nacional "Dr. Juan Tanca Marengo" SOLCA Gua yaquil. Av. Pedro Menéndez Gilbert y calle Atahualpa, Parroquia Tarqui, Guayaquil - Ecuador, Código postal 090505. Teléfono: (593) 3718300 ext. 2464; 997202031

Conflicto de intereses: LoS autores declaran no tener conflictos de intereses.

Recibido: 11 de Enero 2021 Aceptado: 20 de Febrero, 2021 Publicado: 30 de Abril, 2021 Editor: Dr. Felipe Campoverde

\section{Membrete bibliográfico:} Real Cotto J, Tanca-Campozano J, Jaramillo Feijoo L, Puga Peña G, Quinto Briones R. Caracterización epidemiológica de las neoplasias del tejido hematopoyético y linfoide atendidos en el Instituto Oncológico Nacional-SOLCA Guayaquil. Rev. Oncol. Ecu 2021;31(1):46-55.

DOI: https://doi.org/10.33821/544

Copyright Real Cotto J, et al. Este artículo es distribuido bajo los términos de Creative Commons Attribution License, el cual permite el uso y redistribución citando la fuente $y$ al autor original.

\section{Caracterización epidemiológica de las neoplasias del tejido hematopoyético y linfoide atendidos en el Instituto Oncológico Nacional-SOLCA Guayaquil}

\section{Epidemiological characterization of Hematopoietic and Lymphoid Neoplasms treated at the National On- cologicall Institute-SOLCA Guayaquill}

\section{Jhony Joe Real-Cotto*1 (iD, Juan Pablo Tanca-Campozano², Leyda Eli- zabeth Jaramillo-Feijoo1, Gonzalo Rafael Puga-Peña ${ }^{3}$, Rina Mariuxi Quinto-Briones 1}

1. Departamento de Bioestadística, Instituto Oncológico Nacional "Dr. Juan Tanca Marengo", SOLCA Guayaquil.

2. Dirección de Registro de Tumores, Instituto Oncológico Nacional "Dr. Juan Tanca Marengo", SOLCA Guayaquil.

3. Gerencia hospitalaria, Instituto Oncológico Nacional "Dr. Juan Tanca Marengo", SOLCA Guayaquil.

\section{Resumen}

Introducción: Dentro de las cinco primeras neoplasias en el mundo están las Leucemias, la misma que ha venido incrementándose en las últimas décadas. El objetivo del presente estudio fue determinar las características epidemiológicas de las Neoplasias del tejido Hematopoyético y linfoide en pacientes atendidos en el Instituto Oncológico Nacional-SOLCA Guayaquil durante los años 2015 al 2019.

Metodología: Se efectuó un estudio de diseño observacional, descriptivo poblacional; donde el universo y la muestra fueron 891 casos nuevos de neoplasias del Tejido Hematopoyético y Linfoide, recolectándose los datos en una matriz, que fueron tomados del programa informático del registro de tumores.

Resultados: se diagnosticaron Leucemias Linfoideas (69.58\%) y Leucemias Mieloides (30.30\%); más en hombres (53.33\%) que mujeres (44.67\%); siendo los casos en menores de 19 años en Guayaquil del $57.33 \%$ y en Otras ciudades con el $64.36 \%$; en Guayaquil el grupo de edad de 5 - 9 años fue más frecuente con $20.57 \%$ seguido de los menores de 5 años con $19.02 \%$; mientras que en Otras ciudades fueran los menores de 5 años con 20.72\% seguido del grupo etario de 5 - 9 años con 18.33\%; entre otros grupos. Su mayor frecuencia en Guayaquil fueron en parroquias Tarqui, Ximena y en Otras ciudades en Región Costa (81.47\%). 
Conclusión: Dentro de las neoplasias del Tejido Hematopoyético y Linfoide la más común fue las Leucemias Linfoideas en la población menor de 19 años con énfasis en los niños menores de 5 años mostrando una presencia importante en los años de estudio en Guayaquil y en la región Costa del Ecuador.

Palabras claves:

DeCS: Neoplasias hematológicas, sistema hematopoyético; características epidemiológicas; Leucemias, Epidemiología, Investigación sobre Servicios de Salud.

DOI: $10.33821 / 544$

\section{Abstract}

Introduction: Leukemias are among the first five neoplasms in the world, the same one that has been increasing in recent decades. The aim of this study was to determine the epidemiological characteristics of Hematopoietic and Lymphoid Neoplasms in patients treated at the National Oncological InstituteSOLCA Guayaquil during the years 2015 to 2019.

Methodology: An observational, descriptive population study was carried out; Where the universe and the sample were 891 new cases of Hematopoietic and Lymphoid neoplasms, the data was collected in a matrix, taken from the tumor registry computer program.

Results: Lymphoid Leukemias were diagnosed in 69.58\% and Myeloid Leukemias with 30.30\%; more in men (53.33\%) than women (44.67\%); being the cases in minors of 19 years in Guayaquil $57.33 \%$ and in Other cities it had $64.36 \%$; in Guayaquil the age group of 5 - 9 years was more frequent with $20.57 \%$ followed by those under 5 years with 19.02\%; while in Other cities under 5 years old with $20.72 \%$ followed by the age group of 5 - 9 years old with 18.33\%; among other groups. Its highest frequency in Guayaquil was in Tarqui and Ximena parishes and in other cities it was in the Coastal Region with $81.47 \%$.

Conclusion: Among the Hematopoietic and Lymphoid neoplasms the most common was Lymphoid Leukemias in the population under 19 years of age, with emphasis on children under 5 years of age, showing an important presence in the years of study in Guayaquil and in the Ecuadorian Coastal region.

\section{Keywords:}

MESH: Hematologic Neoplasms, Neoplasms, hematopoietic System; Leukemia epidemiological characteristics; Leukemias, Epidemiology, Health Services Research.

DOI: $10.33821 / 544$

\section{Introducción}

El cáncer es un problema importante de salud pública, considerado como la segunda causa de mortalidad en el mundo; su incidencia aumenta y se prevé que se duplique para las próximas dos décadas $[1,2]$. Un grupo importante lo constituyen las neoplasias del tejido hematopoyético y tejidos linfoides, en las que han existido enormes avances en su entendimiento y manejo, por lo que la tradicional conceptualización realizada en el año 2008 por la Organización Mundial de la Salud (OMS) ha tenido que ser revisada y actualizada en el año 2016 [3, 4]. 
Las Leucemias se encuentran entre las cinco neoplasias más frecuentes en el mundo [5], se presentan frecuentemente en la población infantil. Reportes que ponen de manifiesto esta prevalencia a nivel regional han sido publicados en Bolivia, en donde se ha reportado una incidencia en aumento del grupo global de leucemias agudas, en mayor proporción la Leucemia Linfoblástica Aguda (LLA), y en segundo lugar la Leucemia Mieloide Aguda (LMA). Estos resultados parecen repetirse en Latinoamérica, por ejemplo en Colombia, dentro de la caracterización epidemiológica en pacientes pediátricos, la mayoría correspondían a la LLA [] $]$. Sin embargo hay zonas geográficas que reportan a la LMA como la más frecuente, seguido del Linfoma No Hodgkin como lo publicado en el hospital Belén de Trujillo en Perú [7].

En el Ecuador y en Guayaquil no ha estado exenta de esta situación, estando entre las principales causas de morbilidad a nivel oncológico de las neoplasias de precursores, caracterizadas por la proliferación anormal de células inmaduras en la médula ósea; como lo observado en la población atendida en el Instituto Oncológico Nacional (ION) - Sociedad de Lucha Contra el Cáncer en el Ecuador (SOLCA) Guayaquil, por lo que se requiere conocer la afectación de las neoplasias del tejido hematopoyético y linfoide, con el propósito de establecer una línea de base para la prevención y control de estas enfermedades. Siendo el objetivo de esta investigación el determinar las características epidemiológicas de las Neoplasias del Tejido Hematopoyético y Linfoide en pacientes atendidos en el Instituto Oncológico Nacional-SOLCA Guayaquil, durante los años 2015 al 2019.

\section{Materiales y métodos Diseño del estudio \\ El presente estudio es observacional, transversal descriptivo.}

\section{Área de estudio}

El estudio se realizó en el servicio de estadística del Instituto Oncológico Nacional "Dr. Juan Tanca Marengo" de la Sociedad de Lucha contra el Cáncer-SOLCA, Guayaquil Ecuador, la cual es un centro de referencia de entidades oncológicas de la zona 8 de distribución territorial del Ecuador que incluye los cantones de Guayaquil, Sanborondón y Durán. De la zona 5: Provincias de Santa Elena, Guayas, Los Ríos y Galápagos. Con un área de influencia de más de 5 millones de habitantes.

\section{Universo y muestra}

El universo fue conformado por todos los pacientes registrados en la institución con diagnóstico de enfermedades hematopoyéticas. Se utilizó para la identificación el código internacional de enfermedades CIE-10, con los códigos desde C1 hasta C95. El cálculo del tamaño muestral fue no probabilístico [8, 9$]$ tipo censo en donde se incluyeron todos los casos posibles de la institución en las fechas comprendidas entre el 1ro de enero del 2015 hasta el 31 de diciembre del 2019. 


\section{Participantes}

Se incluyeron casos en quienes se registraron el ingreso por primera ocasión a la institución con enfermedades hematopoyéticas con edades comprendidas entre los 0 años hasta la edad máxima registrada en la base de datos.

\section{Variables}

La variables fueron diagnostico morfológico, sexo, grupo etario, tiempo (años) y lugar (procedencia).

\section{Procedimientos, técnicas e instrumentos.}

Los datos fueron recogidos de la historia clínica en un formulario diseñado exclusivamente para tal propósito. Para garantizar la confiabilidad de la información los investigadores fueron entrenados sobre la recolección de los datos.

\section{Análisis estadístico}

Recopilada la información se ingresó en una matriz de datos del software SPSS ${ }^{\mathrm{Tm}} 15.0$ (IBM, Chicago, USA). Se utilizó estadística descriptiva en base a frecuencias y porcentajes para las variables cualitativas y para las cuantitativas medidas de tendencia central.

\section{Resultados}

El estudio incluyó 891 pacientes.

\section{Caracterización demográfica}

En la tabla 1, se observan las neoplasias del Tejido Hematopoyético y Linfoide según diagnóstico morfológico en pacientes atendidos en el Instituto Oncológico Nacional - SOLCA Guayaquil entre los años 2015 a 2019, obteniéndose de manera general que mayormente fueran las Leucemias Linfoideas con 69.58\% de los casos, seguido de Leucemias Mieloides con el 30.30\%; asimismo, en los casos atendidos de Guayaquil y otras ciudades tuvieron el mismo comportamiento de más frecuencia en las Leucemias Linfoides. Las mencionadas Leucemias se las clasificó en agudas y crónicas, de las cuales tanto en Mieloide y Linfoide su presentación aguda es la de mayor proporción, en comparación con las leucemias crónicas; siendo la Leucemia Linfoblástica aguda la más representativa.

Tabla 1 Neoplasias del Tejido Hematopoyético y Linfoide, según diagnóstico morfológico. Período 2015 - 2019

\begin{tabular}{lccccccccc}
\hline \multicolumn{2}{c}{$\begin{array}{c}\text { Diagnóstico } \\
\text { Morfológico }\end{array}$} & \multicolumn{2}{c}{$\begin{array}{c}\text { Casos Guayaquil } \\
\mathrm{n}=389(\%)\end{array}$} & $\begin{array}{c}\text { Otras ciudades } \\
\mathrm{n}=502(\%)\end{array}$ & $\begin{array}{c}\text { Subtotal } \\
\mathrm{n}=891\end{array}$ & $\begin{array}{c}\text { Total } \\
\mathrm{n}=891\end{array}$ & \multirow{2}{*}{$\%$} \\
\hline Leucemia & LM Aguda & 74 & $19.02 \%$ & 91 & $18.13 \%$ & 165 & $18.52 \%$ & 270 & \multirow{2}{*}{$30.30 \%$} \\
Mieloide & LM Crónica & 48 & $12.34 \%$ & 57 & $11.35 \%$ & 105 & $11.78 \%$ & & \\
\hline Leucemias & LL Aguda & 248 & $63.75 \%$ & 337 & $67.13 \%$ & 585 & $65.66 \%$ & 620 & $69.59 \%$ \\
Linfoides & LL Crónica & 19 & $4.88 \%$ & 16 & $3.19 \%$ & 35 & $3.93 \%$ & & \\
\hline Otras Leucemias & 0 & $0.00 \%$ & 1 & $0.20 \%$ & 1 & $0.11 \%$ & 1 & $0.11 \%$ \\
\hline
\end{tabular}

LL: leucemia linfocítica. LM: Leucemia mielocítica

En la tabla 2 , se observan las neoplasias del Tejido Hematopoyético y Linfoide según año del diagnóstico, en pacientes atendidos en el Instituto Oncológico Nacional - SOLCA Guayaquil entre los años 2015 a 2019, de forma general se tuvo que las atenciones fueran más de otras 
ciudades con el $56.34 \%$ comparadas con los pacientes de la ciudad de Guayaquil del $43.66 \%$ en la mayoría de años de estudio.

Tabla 2 Neoplasias del Tejido Hematopoyético y Linfoide según año del diagnóstico, en el Instituto Oncológico Nacional - SOLCA Guayaquil

\begin{tabular}{rccccc}
\hline Año & $\begin{array}{c}\text { Casos Guayaquil } \\
\mathrm{n}=389\end{array}$ & $\%$ & $\begin{array}{c}\text { Otras ciudades } \\
\mathrm{n}=502\end{array}$ & $\%$ & $\begin{array}{c}\text { Total casos } \\
\mathrm{n}=891\end{array}$ \\
\hline 2015 & 105 & 46.26 & 122 & 53.74 & 227 \\
2016 & 47 & 33.81 & 92 & 66.19 & 139 \\
2017 & 79 & 51.97 & 73 & 48.03 & 152 \\
2018 & 61 & 37.89 & 100 & 62.11 & 161 \\
2019 & 97 & 45.75 & 115 & 54.25 & 212 \\
\hline
\end{tabular}

La Tabla $\underline{3}$, se muestran a los pacientes con neoplasias del Tejido Hematopoyético y Linfoide según sexo del Instituto Oncológico Nacional - SOLCA Guayaquil entre los años 2015 a 2019, en el que se obtuvo en hombres el $53.33 \%$ y mujeres el $44.67 \%$. De igual manera se presenta en los casos de Guayaquil y de otras ciudades.

Tabla 3 Neoplasias del Tejido Hematopoyético y Linfoide, según sexo. Período 2015 - 2019.

\begin{tabular}{|c|c|c|c|c|c|c|}
\hline Sexo & $\begin{array}{c}\text { Casos Guayaquil } \\
n=389\end{array}$ & $\%$ & $\begin{array}{c}\text { Otras ciuda- } \\
\text { des } \\
n=502\end{array}$ & $\%$ & $\begin{array}{c}\text { Total, ca- } \\
\text { sos } \\
n=891\end{array}$ & $\%$ \\
\hline Hombre & 210 & $53.98 \%$ & 283 & $56.37 \%$ & 493 & 55.33 \\
\hline Mujer & 179 & $46.02 \%$ & 219 & $43.63 \%$ & 398 & 44.67 \\
\hline
\end{tabular}

En la figura 1, se observan a los pacientes con neoplasias del Tejido Hematopoyético y Linfoide según grupo etario atendidos en el Instituto Oncológico Nacional - SOLCA Guayaquil entre los años 2015 a 2019, en el que muestra que en menores de 19 años en Guayaquil tuvo el $57.33 \%$ de los casos y en los casos atendidos de Otras ciudades tuvo $64.36 \%$. En la ciudad de Guayaquil el grupo de edad de 5 - 9 años fue el más afectado con $20.57 \%$ seguido de los menores de 5 años con 19.02\%; mientras que en Otras ciudades fueran los menores de 5 años con $20.72 \%$ seguido del grupo etario de 5 - 9 años con $18.33 \%$; entre otros grupos.

En la Tabla 4 se observan las neoplasias del Tejido Hematopoyético y Linfoide de acuerdo a la procedencia por parroquias de Guayaquil, entre los años 2015 a 2019, obteniéndose que la parroquia Tarqui fuera la de mayor frecuencia con $37.02 \%$, seguido de la Ximena con $24.16 \%$, Febres Cordero con $14.40 \%$, entre otras parroquias; siendo las parroquias mencionadas las más representativas con el $75.58 \%$. 


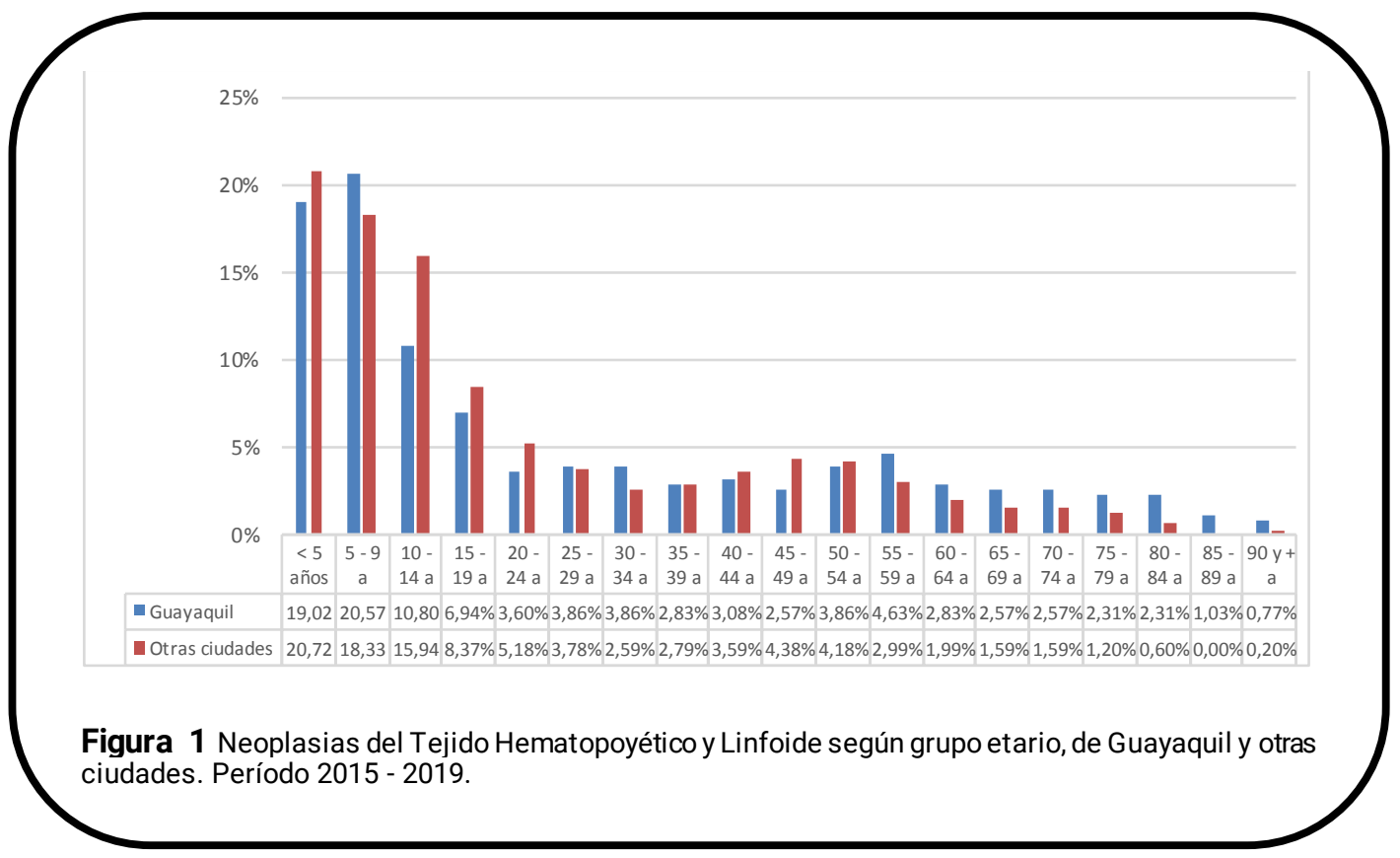

Tabla 4 Neoplasias del Tejido Hematopoyético y Linfoide, según parroquias de Guayaquil. Período 2015 - 2019.

\begin{tabular}{|c|c|c|}
\hline Parroquia & Casos $n=389$ & $\%$ \\
\hline Tarqui & 144 & $37.02 \%$ \\
\hline Ximena & 94 & $24.16 \%$ \\
\hline Febres cordero & 56 & $14.40 \%$ \\
\hline Carbo & 19 & $4.88 \%$ \\
\hline Pascuales & 19 & $4.88 \%$ \\
\hline Bolívar & 18 & $4.63 \%$ \\
\hline Letamendi & 8 & $2.06 \%$ \\
\hline Ayacucho & 4 & $1.03 \%$ \\
\hline García moreno & 3 & $0.77 \%$ \\
\hline Roca & 3 & $0.77 \%$ \\
\hline Chongon & 2 & $0.51 \%$ \\
\hline Juan Gómez Rendón (Progreso) & 2 & $0.51 \%$ \\
\hline Olmedo & 1 & $0.26 \%$ \\
\hline Rocafuerte & 1 & $0.26 \%$ \\
\hline Sucre & 1 & $0.26 \%$ \\
\hline Tenguel & 1 & $0.26 \%$ \\
\hline Guayaquil, cabecera cantonal & 13 & $3.33 \%$ \\
\hline
\end{tabular}

En la Tabla $\underline{5}$, se observan las neoplasias del Tejido Hematopoyético y Linfoide atendidos en el Instituto Oncológico Nacional - SOLCA Guayaquil procedentes de Otras ciudades, según provincias y región, entre los años 2015 a 2019, donde se obtuvo a la provincia del Guayas (excepto Guayaquil) fuera la de mayor frecuencia con el $36.45 \%$, seguido de Los Ríos con 15.94\%, Manabí con 10.16\%, ente otras provincias. En cuanto a los casos atendidos según Región se observó que mayormente eran de la Costa con $81.47 \%$, seguido de la Sierra con $16.94 \%$ de las Regiones de Oriente e Insular con $0.60 \%$ respectivamente. Cabe indicar que el mayor peso según la procedencia la tiene el área geográfica de influencia de la Institución. 
Tabla 5 Neoplasias del Tejido Hematopoyético y Linfoide, según sexo. Período 2015 - 2019.

\begin{tabular}{|c|c|c|c|c|}
\hline Región & 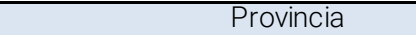 & Casos & $\%$ & \% Región \\
\hline \multirow{7}{*}{ Costa } & Guayas (otros cantones) & 183 & $36.45 \%$ & \multirow{7}{*}{$81.47 \%$} \\
\hline & Los Ríos & 80 & $15.94 \%$ & \\
\hline & Manabí & 51 & $10.16 \%$ & \\
\hline & Santa Elena & 38 & $7.57 \%$ & \\
\hline & El Oro & 35 & $6.97 \%$ & \\
\hline & Esmeraldas & 12 & $2.39 \%$ & \\
\hline & Santo Domingo de los Tsáchilas & 10 & $1.99 \%$ & \\
\hline \multirow{10}{*}{ Sierra } & Pichincha & 25 & $4.98 \%$ & \multirow{10}{*}{$16.94 \%$} \\
\hline & Azuay & 17 & $3.39 \%$ & \\
\hline & Cañar & 11 & $2.19 \%$ & \\
\hline & Chimborazo & 10 & $1.99 \%$ & \\
\hline & Bolívar & 7 & $1.39 \%$ & \\
\hline & Carchi & 4 & $0.80 \%$ & \\
\hline & Tungurahua & 4 & $0.80 \%$ & \\
\hline & Cotopaxi & 3 & $0.60 \%$ & \\
\hline & Loja & 3 & $0.60 \%$ & \\
\hline & Imbabura & 1 & $0.20 \%$ & \\
\hline \multirow{3}{*}{ Oriente } & Pastaza & 1 & $0.20 \%$ & \multirow{3}{*}{$0.60 \%$} \\
\hline & Sucumbíos & 1 & $0.20 \%$ & \\
\hline & Zamora Chinchipe & 1 & $0.20 \%$ & \\
\hline Insular & Galápagos & 3 & $0.60 \%$ & $0.60 \%$ \\
\hline No definido & Desconocida & 2 & $0.40 \%$ & \\
\hline Total & & 502 & $100.00 \%$ & \\
\hline
\end{tabular}

\section{Discusión}

El estudio de caracterización de los pacientes con leucemia aguda en Quito, obtuvo que mayormente fue la leucemia mieloide aguda con el $62.30 \%$; Las leucemias agudas fueron más común en hombres con el $54.45 \%$ que en mujeres; donde la leucemia mieloide aguda fue el tipo más común en la población adulta [10]; siendo este estudio diferente en cuanto al tipo de leucemia más frecuente con respecto a Guayaquil.

Según el estudio del perfil clínico epidemiológico en pacientes pediátricos con cáncer linfohematopoyético en Piura, se demuestra que el cáncer más frecuente fue la leucemia linfoblástica aguda seguido de linfoma No Hodgkin; de ellos el 56\% fueron mujeres, siendo el grupo más afectado en edad escolar en edad promedio de 6 años [11] que al compararlo con esta investigación, aunque el tipo de Leucemias y la edad son muy parecidos, este difiere en el sexo donde es más en hombres.

El estudio de caracterización clínico epidemiológica y supervivencia de pacientes menores de 19 años con leucemia en Cuba, manifiesta que la leucemia es el cáncer más frecuente en la infancia y representa el $41 \%$ de las neoplasias en menores de 15 años; donde determinaron que predominó el grupo etario de 1 a 4 años con $42.7 \%$, en el sexo masculino con el $59 \%$, y la 
leucemia linfoidea aguda como variante morfológica con el 73.2\% [12] siendo este estudio parecido a esta investigación en cuanto a sexo, edad y tipos de neoplasias.

La investigación de hemopatías malignas en Sancti Spíritus en Cuba, en la determinación de la incidencia de neoplasias hematológicas, del total de pacientes en los 5 años de estudio su incidencia fue más predominante en el sexo masculino, con promedio de 37,4 casos por año, el grupo de edad fueron mayores de 60 años; los pacientes con linfoma No Hodgkin tuvieron el $26.7 \%$, seguido de Mieloma Múltiple con 23.6\% y la Leucemia Linfoide crónica con el 12.9\% [2] por lo que este estudio es diferente en cuanto a los tipos de neoplasias y edad de presentación.

En el estudio realizado sobre cáncer infantil del departamento de Antioquia del 2017, para la identificación sobre la incidencia de la LLA en pacientes pediátricos, se obtuvo que el $51.1 \%$ de los casos se presentaron en sexo masculino, asimismo el 50\% de los pacientes tuvieron 8 años o menos, dichos resultados son similares a los encontrados en esta investigación [13].

\section{Conclusiones}

Se concluye que, dentro de las neoplasias del Tejido Hematopoyético y Linfoide, las Leucemias Linfoideas fueron las más comunes en la población menor de 19 años con énfasis en los niños menores de 5 años; así como de las Leucemias Mieloides, en el que han mostrado una presencia importante en los años de estudio en Guayaquil y en la región Costa del Ecuador.

\section{Agradecimientos}

Nota del Editor

La Revista Oncología Ecu permanece neutral con respecto a los reclamos jurisdiccionales en mapas publicados y afiliaciones institucionales.

\section{Información administrativa}

\section{Abreviaturas}

LLA: Leucemia linfocítica aguda. LMA: Leucemia Mielocítica Aguda.

\section{Archivos Adicionales}

Ninguno declarado por los autores. 
Fondos

Los autores no recibieron ningún tipo de reconocimiento económico por este trabajo de investigación.

Disponibilidad de datos y materiales

Existe la disponibilidad de datos bajo solicitud al autor de correspondencia. No se reportan otros materiales.

Contribuciones de los autores

Todos los autores realizaron por igual la conceptualización, curación de datos, análisis formal, adquisición de fondos, investigación, recursos, software, redacción - borrador original, supervisión, validación, visualización, metodología, administración de proyecto, escritura: revisión y edición.

Todos los autores leyeron y aprobaron la versión final del manuscrito.

Aprobación del comité de ética

El protocolo de investigación fue aprobado por el Departamento de Docencia del Hospital Solca - Guayaquil y las autoridades institucionales.

Consentimiento para publicación

El presente estudio es un análisis de base de datos, no aplica para este tipo de estudio.

\section{Referencias}

1. Puyol M, Seoane J, Aguilar E, Vozza LB, Orbe I, Crawford KH, Fernández A, Bray F, Johnson SE, Gopal S. WORLD CANCER RESEARCH DAY: A Call to Action for a Coordinated International Research Effort to Prevent, Diagnose, and Treat Cancer. Clin Cancer Res. 2021 Feb 15;27(4):963-966. doi: 10.1158/1078-0432 CCR-20-2978. Epub 2020 Nov 30. PMID: $\underline{3257425}$

2. Rodríguez-Matos G. Hemopatías malignas en Sancti Spíritus. Estudio de incidencia en 5 años. Rev Cuba Hematol Inmunol Hemoter. 2017;33(S1). SU: revhematologia/697/736

3. Firman G. WHO Classification of Tumors of Hematopoietic and Lymphoid Tissues. WEB Site: MedicalCRITERIA.com. SU: $\underline{\text { medicalcriteria }}$

4. Sorigue M, Sancho J-M. La revisión de 2016 de la clasificación de la OMS de las neoplasias linfoides: la visión del clínico. Med Clínica. 2018;150(2):64-6. doi: 10.1016/i.medcli.2017.04.005

5. Neri Guarachi LE, Torres Aldunate TG. Caracterización epidemiológica, demográfic hematológica de las leucemias mieloides agudas por citometría de flujo en las gestiones 2011-2012 [Thesis]. 2016. SU: umsa.bo/17347

6. Villalba Toquica C del P, Martínez Silva PA, Acero H. Caracterización clínico-epidemiológica de los pacientes pediátricos con leucemias agudas en la Clínica Universitaria Colombia. Serie de casos 2011-2014. Pediatría. 1 de enero de 2016;49(1):17-22. doi:10.1016/j.rcpe.2016.01.002 
7. Temoche P, YordanoR. Características clínico-epidemiológicas y laboratoriales de pacientes adultos hospitalizados con diagnóstico de novo de neoplasias hematológicas. Univ Nac Trujillo [Internet]. 2017; SU: unitru.pe/9450

8. Manterola C, Otzen T. Estudios observacionales: los diseños utilizados con mayor frecuencia en investigación clínica. Int J Morphol. 2014;32(2):634-45. SU: scielo.cl/art42

9. Hernández-Sampieri R, Torres CPM. Metodología de la investigación. Vol. 4. McGraw-Hill Interamericana México^ eD. FDF; 2018.

10. Pérez T del RG, Espín JIP, Terán RNT. Caracterización de los pacientes con leucemia aguda en un hospital de tercer nivel de Quito - Ecuador. Revista Médica-Científica CAMbios HECAM, 18(2), 24-31. doi: 10.36015/cambios.v18.n2.2019.535

11. Zapata M, Pilar F del. Perfil clínico - hematológico y epidemiológico en los pacientes pediátricos con cáncer linfohematopoyético del Servicio de Pediatría en el Hospital III Essalud José Cayetano Heredia - Piura, 2014- 2018. Univ Nac Piura UNP [Internet]. 2019 [citado 24 de febrero de 2021]; SU: unp.pe/1640

12. Querol Betancourt N, Chávez Isla MI, Leblanch Fernández CC, Jimenez Portuondo N, Querol Betancourt N, Chávez Isla MI, et al. Caracterización clinicoepidemiológica y supervivencia de pacientes menores de 19 años con leucemia. MEDISAN. febrero de 2021;25(1):26-40.

13. Gómez-Mercado CA, Segura-Cardona AM, Pájaro-Cantillo DE, Mesa-Largo M. Incidencia y determinantes demográficos de la leucemia linfoide aguda en pacientes con cáncer pediátrico, Antioquia. Univ Salud. 1 de mayo de 2020;22(2):112-9. SU: scielo.co/2389-7066 